\title{
Silicon strip tracking detector development and prototyping for the Phase-II Upgrade of the ATLAS experiment
}

\author{
S. Kuehn on behalf of the ATLAS Collaboration ${ }^{\mathrm{a}, *}$ \\ ${ }^{a}$ Institute of Physics, Albert-Ludwigs-University Freiburg, Freiburg, Germany
}

\begin{abstract}
In about ten years from now, the Phase-II upgrade of the LHC will be carried out. Due to increased luminosity, a severe radiation dose and high particle rates will occur for the experiments. In consequence, several detector components will have to be upgraded. In the ATLAS experiment, the current inner detector will be replaced by an all-silicon tracking detector with the goal of at least delivering the present detector performance also in the harsh Phase-II LHC conditions. This report presents the current planning and results from first prototype measurements of the upgrade silicon strip tracking detector.
\end{abstract}

Keywords: Tracking detectors, Solid-state detectors, Silicon strip detectors, HL-LHC

PACS: 29.40.Cs, 29.40.Wk

\section{Introduction}

The current silicon strip tracker of the ATLAS experiment is performing very well [1]. In about 10 years, the upgrade of the LHC to the HL-LHC will be carried out. This results in an increase of the luminosity and will allow to reach multiple physics goals with an integrated luminosity of $3000 \mathrm{fb}^{-1}$. The Higgs boson properties are expected to be measured with $2-10 \%$ precision. And the mass reach for new physics is expected to be increased by $30-50 \%$ compared to that obtained with an integrated luminosity of $300 \mathrm{fb}^{-1}$ [2]. However, the increased luminosity results in a high radiation damage, high rate and occupancy. Fluences of up to $2 \times 10^{16} 1 \mathrm{MeV} n_{\mathrm{eq}} \mathrm{cm}^{-2}$ may be reached for the inner detector of the ATLAS experiment. In order to keep the performance of the current tracker, a replacement of the inner detector is required. Manifold R\&D is ongoing for prototyping and layouting the new silicon strip tracker.

\section{The new inner tracker: ITK}

The new inner tracker is forseen to consist of a pixel and strip silicon tracking detector [3]. The baseline layout has four layers of pixel detectors in the barrel region and six in the forward region. The strip detector is planned to have five barrel and seven endcap layers. In total these sum up to $200 \mathrm{~m}^{2}$ of silicon with more than 100 million channels. The layout is shown in Fig. 1. Requirements are radiation hardness, high granularity and low material budget. Moreover, the implementation in the existing detector, cost and mass production has to be kept in

\footnotetext{
${ }^{*}$ Corresponding author

Email address: susanne.kuehn@cern.ch (S. Kuehn on behalf of the ATLAS Collaboration)
}

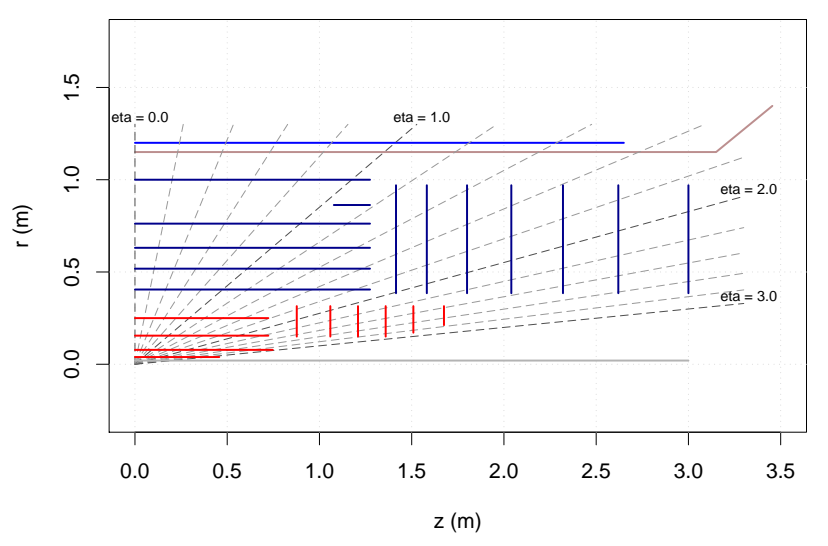

Figure 1: The baseline layout of the upgraded ATLAS inner tracking detector showing the active areas of silicon detectors arranged on cylinders and disks.

mind. To achieve the multiplexing of services, fast data transmission with low power giga-bit transmitters, cooling with $\mathrm{CO}_{2}$ and triggering at Level 0 with $1 \mathrm{MHz}$ rate are deployed. Currently, a detailed study of the benefits for the physics reach of a possible increase of acceptance to $|\eta|$ around 4 is ongoing.

\subsection{The upgrade silicon strip tracker}

The silicon strip tracker for the upgrade has the design concept of modularity and high integration. This means single units, like e.g. modules are built and tested standalone and assembled in larger structures afterwards. The modularity aims for easier final assembly, multiple site production, and early system tests. The baseline sensors are n-in-p float zone silicon sensors with a thickness of around $320 \mu \mathrm{m}$. They are built into modules by gluing on top binary readout electronics which are based on $130 \mathrm{~nm}$ CMOS ASICs. The modules are single-sided 
(a)

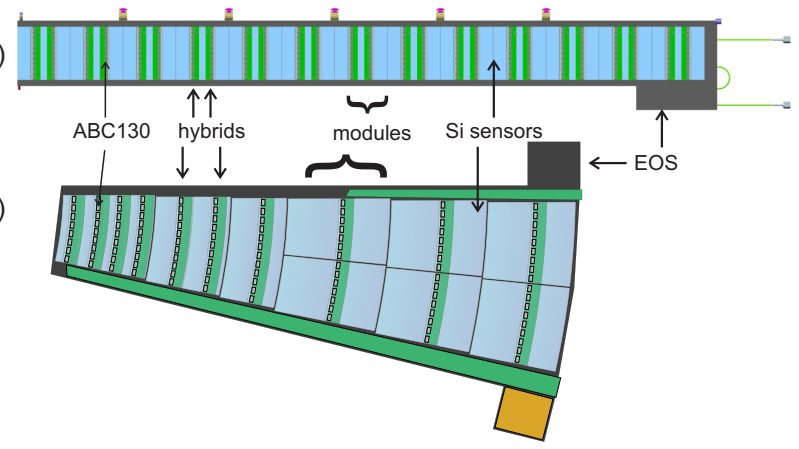

Figure 2: a) Barrel stave components, b) Petal components.

objects glued with 40 mrad stereo angle onto both sides of carbon and cooling structures, so-called staves (for the barrel region) and petals (for the forward region). They are mounted on support structures which include services integrated into panels. The baseline powering scheme is based on DCDC conversion. A sketch of the stave and petal layouts is given in Fig. 2.

\subsection{Developments and prototyping of combined objects}

The prototyping is advanced and several integrated objects have been built. The prototyping of the forward region is pursued in the so-called Petalet project [4]. The project covers the production, assembly and test of two readout layouts on smaller scale than a full petal. Silicon sensors from CNM [5] are attached to readout electronics with $250 \mathrm{~nm}$ CMOS ASICs. About 40 of these modules have been successfully built. Test results show noise values, the figure of merit, of modules for the forward region of noise equivalent to 600-700 electrons (ENC). Tests were performed at a bias voltage of $150 \mathrm{~V}$ at room temperature. Carbon cores were also developed and in total nine cores have been produced for assembly with modules. An example of a petalet is shown in Fig. 3. It consists of a carbon core assembled with bustape for routing power and data. Lower and upper modules and readout boards are glued onto the core surface. Moreover, prototyping of full-size objects in ongoing [6]. Full-size staves have been built and successfully tested including both serially and DCDC powered staves with $1.3 \mathrm{~m}$ length. The input noise at $1 \mathrm{fC}$ is $600-670 \mathrm{ENC}$ for a DCDC powered stave demonstrating overall a very good performance. For the forward region, a thermo-mechanical petal is in preparation.

\subsection{Results for sensors and new readout ASICs}

For qualifying new silicon sensor material a large study was conducted. Several hundred n-in-p float zone mini sensors from HPK [7] were tested. In extensive tests of sensors irradiated with neutrons, gammas or protons to fluences of up to $2 \times 10^{15} 1 \mathrm{MeV} n_{\mathrm{eq}} \mathrm{cm}^{-2}$ their radiation hardness could be demonstrated. At the highest fluence the collected charge is above 8000 electrons at a bias voltage of $500 \mathrm{~V}$.

Additionally, new binary ASICs have been developed in $130 \mathrm{~nm}$ CMOS technology. Initial tests show the expected performance. More hybrids will be assembled soon. Data taking

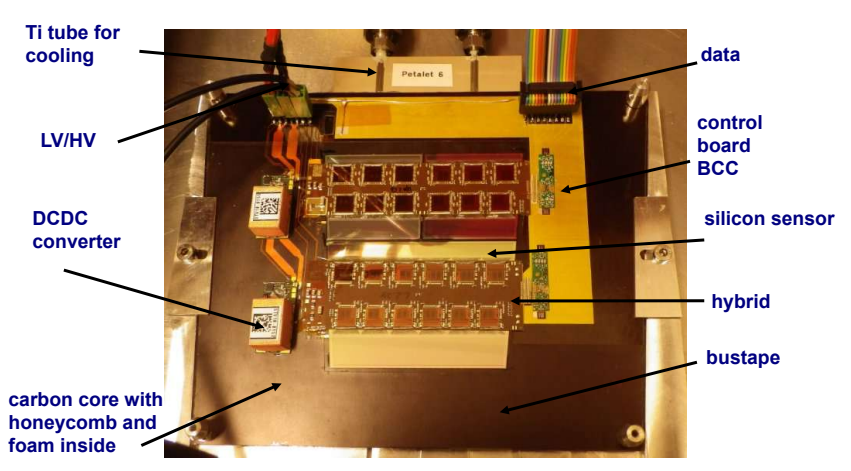

Figure 3: Photo of a petalet. It consists of a carbon core with bustape on top. Two silicon detector modules and readout boards are glued on the bustape.

in a test beam has been conducted recently and the analysis of the data is ongoing.

\section{Conclusion}

A new all-silicon tracker is foreseen for the Phase-II upgrade of the ATLAS experiment. The development and prototyping programme for the new silicon strip detector system is well advanced. About 70 barrel and 40 endcap prototype modules have been produced with well controlled production and module properties. Electrical measurements show low noise values. Larger structures, like staves and petalets have been assembled and successfully tested. Full-size prototyping is approaching maturity. A sensor study with n-in-p strip sensors shows that they withstand doses of $2 \times 10^{15} 1 \mathrm{MeV} n_{\mathrm{eq}} \mathrm{cm}^{-2}$. New ASICs have been designed, which show encouraging results in first tests. Many additional R\&D tasks are ongoing and the collaboration is planning for preparing a technical design report until end of 2016.

\section{Acknowledgments}

This work was supported in part by the European Social Fund and by the Ministry Of Science, Research and the Arts BadenWuerttemberg.

\section{References}

[1] The ATLAS Collaboration, The ATLAS Experiment at the CERN Large Hadron Collider, JINST 3 (2013) S08003.

[2] F. Gianotti, RLIUP Workshop, Archamps, October 2013 https://indico.cern.ch/event/260492/session/1/contribution/4/material/slides/1.pdf.

[3] The ATLAS Collaboration, Letter of Intent for Phase-II Upgrade of the ATLAS Experiment, CERN-LHCC-2012-022, LHCC-1-023 (2013).

[4] M. Aliev, et al.,A forward silicon strip system for the ATLAS HL-LHC upgrade, Nucl. Instrum. and Meth. A730 (2013) 210-214.

[5] IMB-CNM, Instituto de Microelectronica de Barcelona, Centro Nacional de Microelectronica, 08193 Cerdanyola del Valles (Bellaterra), Barcelona, Spain.

[6] S. Diez on behalf of the ATLAS Collaboration, Silicon strip staves and petals for the ATLAS upgrade tracker of the HL-LHC, Nucl. Instrum. and Meth. A699 (2013) 93-96.

[7] Hamamatsu Photonics Ltd., http://www.hamamatsu.com. 